\title{
Minerais orgânicos e licopeno na alimentação de poedeiras: desempenho zootécnico e qualidade dos ovos
}

\author{
[Organic minerals and lycopene in the diet of laying hens: production \\ performance and egg quality] \\ E.R.M. Garcia ${ }^{1}$, F.K. Cruz ${ }^{2}$, C. Kiefer ${ }^{3}$, L.R. Avila ${ }^{4}$, R.P.P. Souza ${ }^{4}$ \\ ${ }^{1}$ Universidade Estadual de Mato Grosso do Sul - Aquidauana, MS \\ ${ }^{2}$ Aluna de pós-graduação - Universidade Estadual de Mato Grosso do Sul - Aquidauana, MS \\ ${ }^{3}$ Universidade Federal de Mato Grosso do Sul - Campo Grande, MS \\ ${ }^{4}$ Aluna de graduação - Universidade Estadual de Mato Grosso do Sul - Aquidauana, MS
}

\begin{abstract}
RESUMO
Objetivou-se avaliar o efeito da utilização de minerais orgânicos e do licopeno em rações para poedeiras sobre o desempenho zootécnico e a qualidade dos ovos. Utilizaram-se 288 poedeiras, distribuídas em DIC em esquema fatorial $2 \times 3$ (fontes de minerais x níveis de licopeno), com seis tratamentos, seis repetições e oito aves por unidade experimental. As rações experimentais foram: minerais inorgânicos (MI) sem a adição de licopeno; MI com a adição de licopeno $(400 \mathrm{mg} / \mathrm{kg})$; MI com a adição de licopeno $(800 \mathrm{mg} / \mathrm{kg})$; minerais orgânicos (MOR) sem a adição de licopeno; MOR com a adição de licopeno $(400 \mathrm{mg} / \mathrm{kg})$; MOR com a adição de licopeno $(800 \mathrm{mg} / \mathrm{kg})$. Foram avaliados: consumo de ração, porcentagem de postura, massa dos ovos, conversão alimentar $(\mathrm{kg} / \mathrm{kg}$ e $\mathrm{kg} / \mathrm{dz})$, peso do ovo, porcentagens de casca, albúmen e gema, espessura da casca, gravidade específica, unidade Haugh, índice e coloração de gema, $\mathrm{pH}$ do albúmen e da gema. Os minerais orgânicos aumentam o consumo de ração quando associados a níveis de 0 e $800 \mathrm{mg}$ de licopeno. A associação de $400 \mathrm{mg}$ de licopeno com minerais inorgânicos aumenta o consumo de ração. A adição de minerais orgânicos ou de $400 \mathrm{mg}$ de licopeno às rações melhora a porcentagem de postura e massa dos ovos de poedeiras com 58 semanas de idade. A coloração de gema é mais acentuada para as fontes inorgânicas em relação às orgânicas e mais acentuada em rações com 800mg de licopeno. A unidade Haugh é maior em rações sem licopeno e com minerais inorgânicos e em rações com 400mg de licopeno e com minerais orgânicos. Rações com fonte orgânica associada a $800 \mathrm{mg}$ de licopeno proporcionam maior unidade Haugh em relação a fonte orgânica sem licopeno.
\end{abstract}

Palavras-chave: antioxidante natural, carotenoide, consumo de ração, cor, unidade Haugh

\begin{abstract}
This study aimed to evaluate the effect of the use of organic minerals and lycopene in the diet of laying hens on performance and egg quality. 288 hens were used, distributed in DIC in a factorial $2 \times 3$ (mineral sources $x$ lycopene levels) with six treatments and six replicates of eight birds per experimental unit. The experimental diets were: inorganic minerals (MI) without the addition of lycopene; MI with the addition of lycopene (400mg/kg); MI with the addition of lycopene $(800 \mathrm{mg} / \mathrm{kg}) ;$ minerals organic (MOR) without addition lycopene; MOR with the addition of lycopene $(400 \mathrm{mg} / \mathrm{kg}) ; \mathrm{MOR}$ with the addition of lycopene $(800 \mathrm{mg} / \mathrm{kg})$. The following parameters were evaluated: feed intake, egg production, egg mass, feed conversion ( $\mathrm{kg} / \mathrm{kg}$ and $\mathrm{kg} / \mathrm{dz})$, egg weight, percentage shell, albumen and yolk, shell thickness specific gravity, unit Haugh, index and yolk color, $\mathrm{pH}$ of albumen and yolk. The organic minerals increase the feed intake when combined at levels of 0 and $800 \mathrm{mg}$ of lycopene. The combination of $400 \mathrm{mg}$ of lycopene with inorganic minerals increases feed intake. The addition of organic or mineral 400mg lycopene in diets improves egg production and egg weight of hens at 58 weeks of age. The yolk color is more
\end{abstract}

Recebido em 30 de abril de 2015

Aceito em 11 de junho de 2015

E-mail: ermgarcia@uems.br 


\section{Garcia et al.}

pronounced for inorganic sources in relation to organic and more pronounced in diets with $800 \mathrm{mg}$ of lycopene. The Haugh unit is higher in lycopene free diets and diets with inorganic mineral and 400mg of lycopene and organic minerals. Diets with organic source associated with $800 \mathrm{mg}$ of lycopene provide higher Haugh units for organic source without lycopene.

Keywords: natural antioxidant, carotenoid, feed intake, color, Haugh unit

\section{INTRODUÇ̃̃̃O}

Para se obter uma boa nutrição, é necessário que o animal receba quantidades adequadas de nutrientes, incluindo-se os minerais, que são considerados de grande importância para as aves por participarem de todos os processos bioquímicos corporais.

Atualmente no mercado encontram-se disponíveis fontes minerais nas formas orgânicas e inorgânicas; no entanto, as utilizadas nas rações de poedeiras são geralmente oriundas de compostos inorgânicos. Devido à falta de conhecimento relacionada à absorção e utilização desses minerais, os níveis fornecidos nas dietas são frequentemente superiores aos exigidos, resultando em excesso de fornecimento e consequentemente maior excreção ao meio ambiente.

Alguns trabalhos têm demonstrado que a substituição total dos minerais na forma inorgânica por orgânica pode resultar em melhoria no desempenho e qualidade dos ovos das poedeiras. Entretanto, os resultados obtidos na literatura ainda são controversos (Mabe et al., 2003; Sechinato et al., 2006; Maciel et al., 2010).

O ovo apresenta alto conteúdo de ácidos graxos insaturados, que o torna mais propenso à oxidação lipídica. Dessa forma, vários estudos têm sido realizados utilizando antioxidantes, como, por exemplo, minerais, vitaminas, carotenoides (beta-caroteno, licopeno e luteína), além de outros. Entre os minerais destacam-se o zinco $(\mathrm{Zn})$, cobre $(\mathrm{Cu})$, selênio (Se), manganês $(\mathrm{Mn})$ e ferro $(\mathrm{Fe})$, que atuam como cofatores de enzimas, que reduzem os metabólicos reativos de oxigênio (MRO), como glutationa peroxidase (Se), superóxido-dismutase $(\mathrm{Zn}, \mathrm{Cu}$ e $\mathrm{Mn})$ e catalase (Fe) (Ferreira e Matsubara, 1997).

O licopeno faz parte do grupo dos carotenoides e, além de pigmentante, possui função sequestrante de radicais livres (radicais peróxidos e oxigênio molecular), apresentando dessa forma ação antioxidante, bloqueando os radicais livres que danificam as membranas lipoproteicas. Tais mecanismos antioxidantes são de extrema importância no sistema imune e na saúde dos animais (Olson, 1999).

Acredita-se que a associação de antioxidantes possa potencializar sua ação antioxidativa, proporcionando melhoria no desempenho e qualidade dos ovos (Halliwell et al., 2000; Sahin et al., 2006; Ajakaiye et al., 2011). No entanto, ainda não existem informações concretas sobre os possíveis efeitos antioxidantes do licopeno na alimentação de poedeiras. Diante disso, este trabalho foi desenvolvido com o objetivo de avaliar o efeito da adição de minerais orgânicos e do licopeno nas rações sobre o desempenho e a qualidade dos ovos de poedeiras comerciais.

\section{MATERIAL E MÉTODOS}

O experimento foi aprovado pelo Comitê de Ética no Uso de Animais/UEMS, sob protocolo número 018/2013. Utilizaram-se 288 poedeiras comerciais da linhagem Dekalb Brown (poedeiras de ovos vermelhos), com 58 semanas de idade, durante o período de 16 semanas. As aves foram alojadas duas a duas, em gaiolas de arame galvanizado com quatro divisões de $25 \mathrm{x}$ $40 \times 45 \mathrm{~cm}$, em galpão convencional de postura com cobertura de telhas de fibrocimento.

A ração e a água foram fornecidas ad libitum e as aves foram selecionadas por peso (média $\pm 10 \%$ ) e produção de ovos antes do início do experimento, e submetidas a 10 dias de adaptação às dietas experimentais.

O programa de iluminação utilizado foi de 17 horas por dia (iluminação natural + artificial). Durante todo o período experimental foram averiguadas as condições térmicas do aviário por meio das temperaturas máxima e mínima e a umidade relativa do ar, correspondendo às médias de $33,4^{\circ} \mathrm{C} \pm 5,1^{\circ} \mathrm{C}, 21,8^{\circ} \mathrm{C} \pm 4,3^{\circ} \mathrm{C}$ e $63,4 \% \pm 17,9 \%$, respectivamente. 
As aves foram distribuídas em um delineamento inteiramente ao acaso, em esquema fatorial $2 \times 3$ (fontes de minerais $\mathrm{x}$ níveis de licopeno), com seis tratamentos, seis repetições e oito aves por unidade experimental.

As rações experimentais (Tab. 1) foram formuladas para o atendimento das exigências nutricionais da linhagem e a composição química dos alimentos de acordo com o Manual de Criação da Linhagem Dekalb Brown (Granja Planalto, 2009) e Rostagno et al. (2005). Estas foram distribuídas da seguinte forma: MI sem a adição de licopeno; MI com a adição de licopeno (400mg/kg); MI com a adição de licopeno (800mg/kg); MOR sem a adição de licopeno; MOR com a adição de licopeno $(400 \mathrm{mg} / \mathrm{kg})$; MOR com a adição de licopeno (800mg/kg).

Tabela 1. Composições percentual e calculada das rações experimentais

\begin{tabular}{|c|c|c|c|c|c|c|}
\hline Ingredientes & MI & $\begin{array}{c}\text { MI + Lic } \\
(400 \mathrm{mg} / \mathrm{kg})\end{array}$ & $\begin{array}{c}\text { MI + Lic } \\
(800 \mathrm{mg} / \mathrm{kg})\end{array}$ & $\mathrm{MO}$ & $\begin{array}{l}\mathrm{MO}+\mathrm{Lic} \\
(400 \mathrm{mg} / \mathrm{kg})\end{array}$ & $\begin{array}{c}\mathrm{MO}+\mathrm{Lic} \\
(800 \mathrm{mg} / \mathrm{kg})\end{array}$ \\
\hline Milho, grão & 61,49 & 61,49 & 61,49 & 61,49 & 61,49 & 61,49 \\
\hline Farelo de soja, $45 \%$ & 25,27 & 25,27 & 25,27 & 25,27 & 25,27 & 25,27 \\
\hline Óleo de soja & 1,25 & 1,25 & 1,25 & 1,25 & 1,25 & 1,25 \\
\hline Calcário calcítico & 8,27 & 8,27 & 8,27 & 8,27 & 8,27 & 8,27 \\
\hline Fosfato bicálcico & 2,08 & 2,08 & 2,08 & 2,08 & 2,08 & 2,08 \\
\hline L-lisina $\mathrm{HCl}$ & 0,11 & 0,11 & 0,11 & 0,11 & 0,11 & 0,11 \\
\hline DL-metionina & 0,17 & 0,17 & 0,17 & 0,17 & 0,17 & 0,17 \\
\hline Sal comum & 0,35 & 0,35 & 0,35 & 0,35 & 0,35 & 0,35 \\
\hline $\begin{array}{l}\text { Suplemento mineral e } \\
\text { vitamínico* }\end{array}$ & 0,10 & 0,10 & 0,10 & 0,30 & 0,30 & 0,30 \\
\hline Inerte & 0,90 & 0,60 & 0,20 & 0,70 & 0,40 & 0,00 \\
\hline Licopeno** & 0,00 & 0,30 & 0,70 & 0,00 & 0,30 & 0,70 \\
\hline BHT & 0,01 & 0,01 & 0,01 & 0,01 & 0,01 & 0,01 \\
\hline Total & 100,00 & 100,00 & 100,00 & 100,00 & 100,00 & 100,00 \\
\hline \multicolumn{7}{|l|}{ Valores calculados } \\
\hline EM (kcal/kg) & 2.800 & 2.800 & 2.800 & 2.800 & 2.800 & 2.800 \\
\hline PB $(\%)$ & 17,0 & 17,0 & 17,0 & 17,0 & 17,0 & 17,0 \\
\hline $\begin{array}{l}\text { Metionina + cistina } \\
\text { digestíveis }(\%)\end{array}$ & 0,65 & 0,65 & 0,65 & 0,65 & 0,65 & 0,65 \\
\hline Lisina digestível (\%) & 0,85 & 0,85 & 0,85 & 0,85 & 0,85 & 0,85 \\
\hline Cálcio (\%) & 4,10 & 4,10 & 4,10 & 4,10 & 4,10 & 4,10 \\
\hline Fósforo disponível (\%) & 0,48 & 0,48 & 0,48 & 0,48 & 0,48 & 0,48 \\
\hline Ácido linoleico (\%) & 1,79 & 1,79 & 1,79 & 1,79 & 1,79 & 1,79 \\
\hline
\end{tabular}

MI: mineral inorgânico; MO: mineral orgânico (composição: $\mathrm{Cu}, 10 \%$; Fe, 6\%; Mn, 8\%; Se, 0,2\%; Zn, 10\%); Lic: licopeno; *Composição por kg de ração: Vitamina A, 7.000 UI; Vitamina D3, 1.600 UI; Vitamina E, 8 UI; Vitamina K3, 1,0mg; Ácido nicotínico, 20mg; Ácido pantotênico, 7mg; Vitamina $\mathrm{B}_{6}, 1,0 \mathrm{mg}$; Vitamina $\mathrm{B}_{12}, 0,010 \mathrm{mg}$; Biotina, 0,02mg; Cu, 10mg; Fe, 50mg; I, 0,83mg; Mn, 65mg; Se, 0,30mg; Zn, 60mg; **Produto comercial baseado em licopeno puro e tomate em pó para o atendimento de 400 e $800 \mathrm{mg}$ de licopeno/kg de ração (Vetscience Nutracêuticos Ltda.).

Os minerais utilizados na forma orgânica foram: $\mathrm{Cu}, \mathrm{Fe}, \mathrm{Mn}, \mathrm{Zn}$ (complexo metal - aminoácido) e Se (selênio levedura). Os níveis de inclusão para as diferentes fontes de minerais (inorgânica e orgânica) foram diferentes, considerando em ambos os casos o atendimento das exigências de todos os minerais.

Ao $28^{\circ}$ dia de cada ciclo, foram determinados os parâmetros produtivos: consumo de ração (CR), porcentagem de postura (PP), massa dos ovos
(MO) e conversão alimentar (CA) (kg/kg e $\mathrm{kg} / \mathrm{dz})$.

O consumo de ração foi registrado semanalmente e calculado por meio da diferença entre a quantidade de ração fornecida e as sobras ao término de cada semana que compôs o ciclo. A produção de ovos foi registrada diariamente com duas coletas diárias (períodos matutino e vespertino). A massa de ovos foi obtida por meio da multiplicação do peso médio dos ovos pela 
porcentagem de produção dos ovos e expressa em gramas. A conversão alimentar foi calculada por meio do consumo médio de ração $(\mathrm{kg})$ dividido por quilos de ovos (conversão $\mathrm{kg} / \mathrm{kg}$ ) e por dúzia de ovos (conversão kg/dz).

Nos últimos três dias de cada ciclo, foram determinados o peso médio dos ovos (PO), a gravidade específica (GE), as porcentagens de casca (PC), albúmen (PA) e gema (PG), o pH do albúmen (pHa) e da gema (pHg), a unidade Haugh (UH), o índice de gema (IG), a coloração de gema (CG) e a espessura da casca (EC).

Com base nos dados de peso total e número de ovos de cada unidade experimental, determinouse o PO. Após pesados, os ovos foram mergulhados em solução salina com diferentes densidades, variando de 1,070 até 1,098. A técnica foi baseada no princípio da flutuação, em que os ovos foram imersos em recipiente contendo soluções salinas em ordem crescente de densidade. Considerou-se a densidade do ovo aquela em que o mesmo flutuasse.

Foram utilizados três ovos para avaliar as PC, PA, PG, pHa e pHg, e três foram destinados para as análises de qualidade interna. Para a avaliação da qualidade interna, os ovos colhidos foram pesados separadamente em balança semianalítica $( \pm 0,001 \mathrm{~g})$ e quebrados em superfície plana e lisa de vidro.

Com o auxílio de um paquímetro digital, as medidas de altura do albúmen e da gema foram determinadas e expressas em milímetros $(\mathrm{mm})$. Por meio da medida da altura de albúmen $(\mathrm{mm})$ e peso unitário do ovo $(\mathrm{g})$, foram calculados os valores da UH determinada pela equação descrita por Silversides e Budgell (2004): $\mathrm{UH}=100 \mathrm{log}$ $\left(\mathrm{H}+7,75-1,7 \mathrm{~W}^{0,37}\right)$, em que $\mathrm{H}=$ altura do albúmen (mm) e $\mathrm{W}=$ peso do ovo $(\mathrm{g})$. Posteriormente, com o auxílio de um paquímetro manual $( \pm 0,05 \mathrm{~mm})$, foi mensurado o diâmetro da gema e, com base na média dos valores obtidos, calculou-se o IG (altura/diâmetro).

A análise de CG foi efetuada por meio de um leque colorimétrico DSM (Yolk Color Fan ${ }^{\circledR}$ ). Em seguida, as cascas foram lavadas em água corrente, secas à temperatura ambiente por um período de 48 horas e, em seguida, pesadas em balança analítica para a obtenção da PC. Com o auxílio de um micrômetro digital, determinou-se a EC (mm).

Os dados foram submetidos à análise de variância e, para a comparação entre as médias, foi utilizado o Teste Tukey $(\mathrm{P}<0,05)$.

\section{RESULTADOS E DISCUSSÃO}

Houve interação $(\mathrm{P}<0,05)$ entre a fonte mineral e o nível de licopeno para o CR (Tab. 2), demonstrando que a inclusão de MOR nas rações contendo 0 e $800 \mathrm{mg}$ de licopeno promoveu maior CR das aves quando comparada à fonte inorgânica associada a 0 e $800 \mathrm{mg}$ de licopeno (Tab. 3). Por outro lado, quando a fonte mineral utilizada foi inorgânica com a associação de 400mg de licopeno, o CR das aves foi similar ao daquelas que receberam fonte orgânica com $400 \mathrm{mg}$ de licopeno. Constatou-se também que o nível de 400mg de licopeno proporcionou maior $(\mathrm{P}<0,05) \mathrm{CR}$ das aves quando comparado aos níveis de 0 e $800 \mathrm{mg}$ de licopeno nas rações formuladas com MI. Os níveis de licopeno não influenciaram $(\mathrm{P}>0,05) \quad \mathrm{O} \quad \mathrm{CR}$ das aves alimentadas com a ração contendo MOR (Tab. 3).

Esses resultados podem estar relacionados à atuação de alguns MOR (Cu, Fe, Zn, Se e Mn) como cofatores de enzimas antioxidantes e do licopeno que, de forma sinérgica, proporcionaram melhoria do status antioxidante dos animais, refletindo assim em maior CR.

Avaliando a PP e a MO, observou-se efeito isolado das diferentes fontes de minerais e dos níveis de licopeno estudados $(\mathrm{P}<0,01)$, de forma que a utilização do MOR ou a adição de 400mg de licopeno/kg de ração apresentaram os melhores valores para essas variáveis, provavelmente em função do maior $\mathrm{CR}$ observado para as aves desses tratamentos.

No entanto, a utilização de 0 e $800 \mathrm{mg}$ de licopeno/kg de ração proporcionou $(\mathrm{P}>0,05)$ valores de PP e MO similares. É provável que esse resultado esteja relacionado com a saturação nos mecanismos de absorção intestinal promovida pela adição de $800 \mathrm{mg}$ de licopeno na dieta de poedeiras, resultando em baixa eficiência de sua absorção (Olson et al., 2008). 
Minerais orgânicos...

Tabela 2. Consumo de ração (CR), porcentagem de postura (PP), massa dos ovos (MO) e conversão alimentar (CA) de poedeiras alimentadas com diferentes fontes de minerais e níveis de licopeno

\begin{tabular}{|c|c|c|c|c|c|}
\hline Parâmetros & CR (g/ave/dia) & PP $(\%)$ & MO (g/ave) & CA (kg/kg) & $\mathrm{CA}(\mathrm{kg} / \mathrm{dz})$ \\
\hline \multicolumn{6}{|l|}{ Fonte mineral (FM) } \\
\hline Inorgânica & 96,60 & $80,04 \mathrm{~b}$ & $48,87 \mathrm{~b}$ & 1,96 & 1,43 \\
\hline Orgânica & 101,45 & $86,43 \mathrm{a}$ & $52,85 \mathrm{a}$ & 1,93 & 1,41 \\
\hline \multicolumn{6}{|c|}{ Níveis de licopeno (NL) } \\
\hline $0 \mathrm{mg} / \mathrm{kg}$ de ração & 97,72 & $82,11 \mathrm{~b}$ & $49,75 \mathrm{~b}$ & 1,98 & 1,43 \\
\hline $400 \mathrm{mg} / \mathrm{kg}$ de ração & 102,12 & $87,26 \mathrm{a}$ & 53,45 a & 1,90 & 1,40 \\
\hline $800 \mathrm{mg} / \mathrm{kg}$ de ração & 97,22 & $80,34 \mathrm{~b}$ & $49,38 \mathrm{~b}$ & 1,94 & 1,43 \\
\hline Média & 99,02 & 83,24 & 50,86 & 1,94 & 1,42 \\
\hline$\overline{C V}(\%)$ & 5,81 & 7,94 & 8,01 & 4,93 & 5,75 \\
\hline \multicolumn{6}{|l|}{ Valor de $\mathrm{P}$} \\
\hline$\overline{\mathrm{FM}}$ & 0,003 & $<0,001$ & $<0,001$ & 0,333 & 0,430 \\
\hline NL & 0,020 & 0,007 & 0,005 & 0,189 & 0,466 \\
\hline FM x NL & 0,015 & 0,260 & 0,288 & 0,548 & 0,280 \\
\hline
\end{tabular}

Médias seguidas de letras diferentes na coluna diferem entre si pelo Teste Tukey $(\mathrm{P}<0,05)$. CV: coeficiente de variação.

Tabela 3. Consumo de ração (g/ave/dia) de poedeiras alimentadas com diferentes fontes de minerais e níveis de licopeno

\begin{tabular}{lccc}
\hline \multirow{2}{*}{ Fonte mineral } & \multicolumn{3}{c}{ Níveis de licopeno (mg/kg de ração) } \\
\cline { 2 - 4 } & 0 & 400 & 800 \\
\hline Inorgânica & $94,88 \mathrm{Bb}$ & $102,69 \mathrm{~A}$ & $92,23 \mathrm{Bb}$ \\
Orgânica & $100,56 \mathrm{a}$ & 101,56 & $102,22 \mathrm{a}$ \\
\hline
\end{tabular}

Médias seguidas de letras minúsculas diferentes na coluna e médias seguidas de letras maiúsculas diferentes na linha diferem entre si pelo Teste Tukey $(\mathrm{P}<0,05)$.

Esses resultados diferem parcialmente dos encontrados por Sechinato et al. (2006), que não detectaram efeito para as variáveis produtivas de poedeiras em pós pico de produção alimentadas com fontes orgânicas de $\mathrm{Fe}, \mathrm{Zn}, \mathrm{Cu}, \mathrm{Mn}$ e Se, exceto melhoria na PP.

Por outro lado, os dados obtidos no presente estudo corroboram os relatados por Branton et al. (1995) e Paik (2001), que observaram melhoria na PP e na MO de poedeiras alimentadas com MOR quando comparadas às que receberam MI. Segundo os autores, a melhoria no desempenho das poedeiras está relacionada com a maior biodisponibilidade, uma vez que as moléculas dos minerais na forma orgânica não sofrem dissociação em $\mathrm{pH}$ ácido e permanecem com carga elétrica neutra. Dessa forma, são absorvidos e metabolizados mais eficientemente (Miles e Henry, 2000).

Para a variável CA $(\mathrm{kg} / \mathrm{kg}$ e $\mathrm{kg} / \mathrm{dz})$ não se observou efeito $(\mathrm{P}>0,05)$ das fontes minerais $\mathrm{e}$ dos níveis de licopeno estudados, concordando com os resultados obtidos por Sechinato et al. (2006), ao fornecerem $\mathrm{Mn}, \mathrm{Zn}, \mathrm{I}, \mathrm{Se}, \mathrm{Cu}$ e Fe na forma orgânica para poedeiras no período de 48 a 60 semanas de idade.

Ao avaliar a qualidade dos ovos, observou-se interação $(\mathrm{P}<0,01)$ entre a fonte mineral e o nível de licopeno para os valores de UH (Tab. 4), de forma que, quando a fonte mineral utilizada foi a inorgânica, os maiores $(\mathrm{P}<0,05)$ valores de $\mathrm{UH}$ 


\section{Garcia et al.}

foram obtidos sem a adição de licopeno quando comparados à inclusão da fonte inorgânica associada a 400mg de licopeno às rações (Tab. 5). Para a fonte orgânica de minerais, observouse maiores $(\mathrm{P}<0,05)$ valores de $\mathrm{UH}$ quando esta foi associada a $800 \mathrm{mg}$ de licopeno em relação às dietas sem licopeno.

Trabalhos realizados com poedeiras demonstraram que a UH apresenta maiores valores com a suplementação de MOR (Muthusamy e Viswanathan, 1999), concordando parcialmente com os encontrados neste estudo, em que os maiores valores de UH foram obtidos para os ovos de poedeiras que receberam dietas contendo MOR associados a 800mg de licopeno.
Não se observou efeito $(\mathrm{P}>0,05)$ das fontes de minerais e dos níveis de licopeno sobre as variáveis $\mathrm{PO}, \mathrm{IG}, \mathrm{pHa}, \mathrm{pHg}, \mathrm{EC}$ e GE, estando de acordo com Mabe et al. (2003), Chantiraticul et al. (2008) e Swiatkiewicz e Koreleski (2008).

Os resultados demonstraram que, quanto maior a quantidade de licopeno adicionada à ração, mais intensa a $\mathrm{CG}(\mathrm{P}<0,01)$, corroborando os relatados por Olson et al. (2008). Em relação às fontes de minerais, observou-se que a utilização de rações contendo MI aumentou $(\mathrm{P}<0,05)$ a intensidade de $\mathrm{CG}$ dos ovos, discordando dos encontrados na literatura (Pan et al., 2010).

Tabela 4. Peso do ovo (PO), unidade Haugh (UH), índice de gema (IG), coloração de gema (CG), pH do albúmen e da gema, espessura da casca (EC) e gravidade específica (GE) de ovos provenientes de poedeiras alimentadas com diferentes fontes de minerais e níveis de licopeno

\begin{tabular}{|c|c|c|c|c|c|c|c|c|}
\hline \multirow{2}{*}{ Parâmetros } & \multirow{2}{*}{$\begin{array}{l}\mathrm{PO} \\
(\mathrm{g})\end{array}$} & \multirow{2}{*}{ UH } & \multirow{2}{*}{$\begin{array}{c}\mathrm{IG} \\
(\mathrm{mm})\end{array}$} & \multirow{2}{*}{$\mathrm{CG}$} & \multicolumn{2}{|c|}{$\mathrm{pH}$} & \multirow{2}{*}{$\begin{array}{c}\mathrm{EC} \\
(\mathrm{mm})\end{array}$} & \multirow{2}{*}{ GE } \\
\hline & & & & & Albúmen & Gema & & \\
\hline \multicolumn{9}{|l|}{$\begin{array}{l}\text { Fonte mineral } \\
(\mathrm{FM})\end{array}$} \\
\hline Inorgânica & 60,85 & 92,40 & 0,42 & $6,38 \mathrm{a}$ & 7,63 & 6,07 & 0,378 & 1,090 \\
\hline Orgânica & 61,00 & 93,34 & 0,43 & $6,22 \mathrm{~b}$ & 7,61 & 6,07 & 0,379 & 1,090 \\
\hline \multicolumn{9}{|l|}{$\begin{array}{l}\text { Níveis de } \\
\text { licopeno (NL) }\end{array}$} \\
\hline 0mg/kg de ração & 60,51 & 92,70 & 0,42 & $6,07 \mathrm{c}$ & 7,63 & 6,06 & 0,376 & 1,090 \\
\hline $\begin{array}{l}400 \mathrm{mg} / \mathrm{kg} \mathrm{de} \\
\text { ração }\end{array}$ & 61,14 & 92,21 & 0,43 & $6,27 \mathrm{~b}$ & 7,60 & 6,06 & 0,380 & 1,090 \\
\hline $\begin{array}{l}800 \mathrm{mg} / \mathrm{kg} \mathrm{de} \\
\text { ração }\end{array}$ & 61,13 & 93,69 & 0,43 & $6,55 \mathrm{a}$ & 7,63 & 6,08 & 0,380 & 1,090 \\
\hline Média & 60,93 & 92,87 & 0,43 & 6,30 & 7,62 & 6,07 & 0,379 & 1,090 \\
\hline CV (\%) & 2,52 & 2,20 & 1,51 & 4,28 & 1,64 & 1,25 & 3,33 & 0,15 \\
\hline \multicolumn{9}{|l|}{ Valor de $\mathrm{P}$} \\
\hline FM & 0,775 & 0,114 & 0,255 & 0,006 & 0,813 & 0,951 & 0,777 & 0,424 \\
\hline NL & 0,521 & 0,119 & 0,154 & $<0,001$ & 0,868 & 0,850 & 0,649 & 0,759 \\
\hline FM x NL & 0,172 & 0,008 & 0,505 & 0,336 & 0,793 & 0,818 & 0,284 & 0,155 \\
\hline
\end{tabular}

Médias seguidas de letras diferentes na coluna diferem entre si pelo Teste Tukey $(\mathrm{P}<0,05)$. CV: coeficiente de variação.

Tabela 5. Valores de UH de ovos de poedeiras alimentadas com diferentes fontes de minerais e níveis de licopeno

\begin{tabular}{lccc}
\hline \multirow{2}{*}{ Fonte mineral } & \multicolumn{3}{c}{ Níveis de licopeno $(\mathrm{mg} / \mathrm{kg}$ de ração) } \\
\cline { 2 - 4 } & 0 & 400 & 800 \\
\hline Inorgânica & $93,53 \mathrm{~A}$ & $90,66 \mathrm{Bb}$ & $93,01 \mathrm{AB}$ \\
Orgânica & $91,88 \mathrm{~B}$ & $93,76 \mathrm{ABa}$ & $94,38 \mathrm{~A}$
\end{tabular}

Médias seguidas de letras minúsculas diferentes na coluna e médias seguidas de letras maiúsculas diferentes na linha diferem entre si pelo Teste Tukey $(\mathrm{P}<0,05)$. 
As porcentagens de albúmen (PA), gema (PG) e casca (PC) não foram alteradas $(\mathrm{P}>0,05) \mathrm{em}$ função dos tratamentos estudados. Os valores observados para a composição percentual do ovo no presente estudo foram semelhantes aos encontrados na literatura (Garcia et al., 2002; Roberts, 2004; Rocha et al., 2009), de forma que as PA, PG e PC corresponderam a aproximadamente 62,25 e $10 \%$, respectivamente, em relação ao peso do ovo.

\section{CONCLUSÕES}

Os minerais orgânicos aumentam o consumo de ração quando associados a níveis de 0 e $800 \mathrm{mg}$ de licopeno. A associação de 400mg de licopeno com minerais inorgânicos aumenta o consumo de ração. A adição de minerais orgânicos ou de 400mg de licopeno às rações melhora a porcentagem de postura e massa dos ovos de poedeiras com 58 semanas de idade. A coloração de gema é mais acentuada para as fontes inorgânicas em relação às orgânicas e mais acentuada em rações com $800 \mathrm{mg}$ de licopeno. A unidade Haugh é maior em rações sem licopeno e com minerais inorgânicos e em rações com 400mg de licopeno e com minerais orgânicos. Rações com fonte orgânica associada a $800 \mathrm{mg}$ de licopeno proporcionam maior unidade Haugh em relação à fonte orgânica sem licopeno.

\section{AGRADECIMENTOS}

Ao Conselho Nacional de Desenvolvimento Científico e Tecnológico (CNPq) pelo financiamento da pesquisa.

\section{REFERÊNCIAS}

AJAKAIYE, J.J.; CUESTA-MAZORRA, M.; GARCIA-DIAZ, J.R. Vitamins $\mathrm{C}$ and $\mathrm{E}$ can alleviate adverse effects of heat stress on live weight and some egg quality profiles of layer hens. Pakistan Vet. J., v.31, p.30-35, 2011.

BRANTON, S.L.; LOTT, B.D.; MASLIN, W.R.; DAY, E.J. Fatty liver-hemorragic syndrome observed in commercial layers fed diets containing chelated minerals. Avian Dis., v.39, p.631-635, 1995.
CHANTIRATICUL, A.; CHINRASRI, O.; CHANTIRATIKUL, P. Effect of sodium selenite and zinc-L-selenomethionine on performance and selenium concentrations in eggs of laying hens. Asian-Austral. J. Anim. Sci., v.21, p.1048, 2008.

FERREIRA, A.L; MATSUBARA, L.S. Free radicals: concepts, associated diseases, defense system and oxidative stress. Rev. Assoc. Méd. Bras., v.43, p.61-68, 1997.

GARCIA, E.A.; MENDES, A.A.; PIZZOLANTE, C.C. et al. Efeito dos níveis de cantaxantina na dieta sobre o desempenho e qualidade dos ovos de poedeiras comerciais. Braz. J. Poultry Sci., v.4, p.1-7, 2002.

HALLIWELL, B.; ZHAO, K.; WHITEMAN, M. The gastrointestinal tract: a major site of antioxidant activity? Free Radical Res., v.33, p.819-830, 2000.

MABE, I.; RAPP, C.; BAIN, M.M.; NYS, Y. Supplementation of a corn-soybean meal diet with manganese, copper, and zinc from organic or inorganic sources improves eggshell quality in aged laying hens. Poultry Sci., v.82, p.19031913, 2003.

MACIEL, M.P.; SARAIVA, E.P.; AGUIAR, E.F. et al. Effect of using organic microminerals on performance and external quality of eggs of commercial laying hens at the end of laying. Rev. Bras. Zootec., v.39, p.344-348, 2010.

MANUAL de manejo das poedeiras Dekalb Brown. Uberlândia: Granja Planalto, 2009. 39p.

MILES, R.D.; HENRY, P.R. Relative trace mineral bioavailability. Ci. Anim. Bras., v.1, p.73-93, 2000.

MUTHUSAMY, P.; VISWANATHAN, K. Influence of rearing system on the egg quality traits of commercial layers. Indian Vet. J., v.76, p.433-536, 1999.

OLSON, J.A. Carotenoids and human health. Arch. Latinoam. Nutr., v.49, p.7-11, 1999.

OLSON, J.B.; WARD, N.E.; KOUTSOS, E.A. Lycopene incorporation into egg yolk and effects on laying hen immune function. Poultry Sci., v.87, p.2573-2580, 2008. 


\section{Garcia et al.}

PAIK, I. Application of chelated minerals in animal production. Asian-Austral. J. Anim. Sci., v.14, p.191-198, 2001.

PAN, E.A.; RUTZ, F.; DIONELLO, N.J.L. et al. Desempenho de poedeiras semipesadas arraçoadas com a suplementação de selênio orgânico. Rev. Bras. Agrociênc., v.16, p.83-89, 2010.

ROBERTS, J.R. Factors affecting egg internal quality and egg shell quality in laying hens. J. Poulty Sci., v.41, p.161-177, 2004.

ROCHA, T.C.; GOMES, P.C.; DONZELE, J.L. et al. Níveis de lisina digestível em rações para poedeiras no período de 24 a 40 semanas de idade. Rev. Bras. Zootec., v.38, p.1726-1731, 2009.

ROSTAGNO, H.S.; ALBINO, L.F.T.; DONZELLE, J.L. et al. Tabelas brasileiras para aves e suínos: composição de alimentos e exigências nutricionais. 2.ed. Viçosa: UFV, 2005. 186p.
SAHIN, K.; ONDERCI, M.C.; SAHIN, N. et al. Effects of lycopene supplementation on antioxidant status, oxidative stress, performance and carcass characteristics in heat-stressed Japonese quail. J. Thermal Biol., v.31, p.307312, 2006.

SECHINATO, A.S.; ALBUQUERQUE, R.; NAKADA, S. Efeito da suplementação dietética com microminerais orgânicos na produção de galinhas poedeiras. Braz. J. Vet. Res. Anim. Sci., v.43, p.159-166, 2006.

SILVERSIDES, F.G.; BUDGELL, K. The relationships among measures of egg albumen height, pH, and whipping volume. Poultry Sci., v.83, p.1619-1623, 2004.

SWIATKIEWICZ, S.; KORELESKI, J. The effect of zinc and manganese source in the diet for laying hens on eggshell and bones quality. Vet. Med., v.53, p.555-563, 2008. 